\title{
Iterative Calculation of Reflected and Transmitted Acoustic Waves at a Rough Interface
}

\author{
A. P. Berkhoff, P. M. van den Berg, and J. M. Thijssen, Senior Member, IEEE
}

\begin{abstract}
A rigorous iterative technique is described for calculating the acoustic wave reflection and transmission at an irregular interface between two different media. The method is based upon a plane-wave expansion technique in which the acoustic field equations and the radiation condition are satisfied analytically, while the boundary conditions at the interface are satisfied numerically. The latter is accomplished by an iterative minimization of the integrated squared error in the boundary conditions by a conjugate gradient technique, leading to a converging and relatively simple scheme. The plane interface result can be used as starting value. Although in principle the method is rigorous, numerical examples show that in practice there is a lower bound on the error in the boundary conditions which can be achieved.
\end{abstract}

\section{INTRODUCTION}

$\mathbf{R}$ IGOROUS techniques for computing acoustic wave reflection and transmission at a rough interface often are solved by formulating the integral equation for the unknown pressure and particle velocity at the interface [1]-[4]. Discretizing the interface leads to a system of equations from which the pressure and the particle velocity at the interface can be solved [5], [6]. This procedure has the disadvantage that the integral equation is singular, requiring a proper treatment for the diagonal matrix elements if severe numerical errors are to be avoided.

For realistic 3D-simulations the system of equations becomes so large that the solution is beyond the reach of even the present-day's largest computers. Approximate techniques are: the Kirchhoff approximation and the physical optics approximation for short wavelengths. In general, the approximate methods break down if the size of the interface roughness is of the same order as the wavelength.

The method proposed by Rayleigh uses a plane wave expansion and minimizes the error in the approximation of the boundary conditions at the interface [7], [8]. As weighting functions can be used: Dirac-functions, which results in the point-matching method, and exponential functions, which results in the Rayleigh-Fourier method. Although the plane wave expansion is not a valid uniform expansion inside the corrugations of the interface, the method can however be used when the convergence in the mean is taken as point of consideration [7].

A. P. Berkhoff and J. M. Thijssen are with Biophysics Laboratory, Department of Ophthalmology, St.-Radboud University Hospital, P. O. Box 9101, 6500 HB Nijmegen, The Netherlands.

P. M. van den Berg, is with the Center of Technical Geoscience, Laboratory of Electromagnetic Research, Department of Electrical Engineering, Delft University of Technology, P.O. Box 5031, 2600 GA Delft, The Netherlands. IEEE Log Number 9411949.

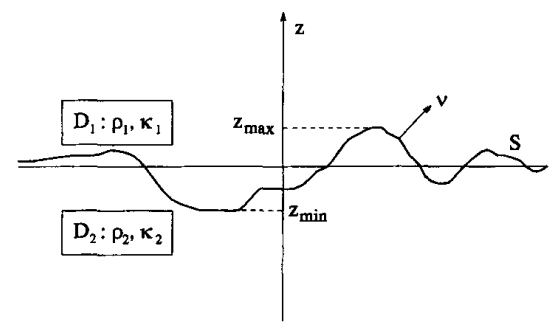

Fig. 1. Definition of the different domains in the configuration.

In this paper, an exact theory for the reflection and transmission of acoustic waves is described where the boundary conditions are satisfied in the mean using an integrated squared error criterion. Meecham's method [9] is also based on an integrated squared error criterion, but his method assumes either rigid periodic surfaces or soft periodic surfaces, i.e., only reflected waves are considered.

The method described in this paper is similar to the one of the electromagnetic wave reflection and transmission problem [10]. The minimization of the integrated squared error is carried out with a conjugate gradient iterative technique. The iterative technique results in minimal storage requirements. The method is valid for media with absorption. However, the complex wavenumber should obey the Karmers-Kronig relations, such as in [11].

\section{Formulation of the Problem}

The configuration for the acoustic reflection and transmission problem is shown in Fig. 1. A point in space is specified by its right-handed, orthogonal coordinates $x, y, z$. It is assumed that the roughness of the interface is a local deformation of an otherwise plane boundary at $z=0$. The analysis is carried out in the temporal frequency domain with angular frequency $\omega$ where the complex time factor $e^{-i \omega t}$ is suppressed. The two fluid like media occupy the domains $D_{1}$ and $D_{2}$, respectively, and are assumed to be linear, homogeneous and isotropic with respective mass densities $\rho_{1}$ and $\rho_{2}$ and compressibilities $\kappa_{1}$ and $\kappa_{2}$. Furthermore, both media exhibit some losses and the real and imaginary parts of $\rho$ and $\kappa$ satisfy the Karmers-Kronig causality relations. The interface is denoted by $S$ and the pressure and the particle velocity vector by $P$ and $\mathrm{V}$, respectively.

In $D_{1}$, a source of finite extent generates a wave incident upon $S$. The incident wave is denoted by $\left\{P_{i}, \mathbf{V}_{i}\right\}$, The total field in $D_{1}$ is written as the superposition of the incident field 
and the reflected field $\left\{P_{r}, \mathbf{V}_{r}\right\}$. The reflected field satisfies the source-free acoustic equations [12]

$$
\begin{aligned}
\nabla \cdot \mathbf{V}_{r}-i \omega \kappa_{1} P_{r} & =0 \\
\nabla P_{r}-i \omega \rho_{1} \mathbf{V}_{r} & =0
\end{aligned} \quad \mathbf{r} \in D_{1}
$$

In these equations $\mathbf{r}=(x, y, z)$ denotes the position vector. The field in $D_{2}$ is denoted as the transmitted field $\left\{P_{t}, \mathbf{V}_{t}\right\}$. It satisfies the source-free acoustic equations

$$
\begin{aligned}
\nabla \cdot \mathbf{V}_{t}-i \omega \kappa_{2} P_{t} & =0 \\
\nabla P_{t}-i \omega \rho_{2} \mathbf{V}_{t} & =0
\end{aligned} \quad \mathbf{r} \in D_{2} .
$$

The fields satisfy the boundary conditions on $S$. At infinity, $\left\{P_{r}, \mathbf{V}_{r}\right\}$ and $\left\{P_{t}, \mathbf{V}_{t}\right\}$ should consist of waves traveling away from $S$. Across $S$ the pressure and the normal components of the particle velocity must be continuous, i.e.,

$$
\begin{array}{cc}
P_{i}+P_{r}=P_{t} & \mathbf{r} \in S \\
\boldsymbol{\nu} \cdot \mathbf{V}_{i}+\boldsymbol{\nu} \cdot \mathbf{V}_{r}=\boldsymbol{\nu} \cdot \mathbf{V}_{t} &
\end{array}
$$

in which $\nu$ is the unit vector in the direction of the normal to $S$, pointing into $D_{1}$.

In the subdomain $z>z_{\max }$ of $D_{1}$, where $z_{\max }$ denotes the maximum value of $z$ on $S$, the reflected field can be written as

$$
\left\{P_{r}, \mathbf{V}_{r}\right\}=\iint_{-\infty}^{\infty}\left\{p_{r}, \mathbf{v}_{r}\right\} e^{i \mathbf{k}_{1}^{+} \cdot \mathbf{r}} d k_{x} d k_{y} \quad\left(z>z_{\max }\right)
$$

in which

$$
\mathbf{k}_{1}^{+}=\left(k_{x}, k_{y},+k_{z, 1}\right)
$$

where

$$
k_{z, 1}=\sqrt{\omega^{2} \rho_{1} \kappa_{1}-k_{x}^{2}-k_{y}^{2}} \mathfrak{R}\left(k_{z, 1}\right), \mathfrak{I}\left(k_{z, 1}\right)>0 .
$$

Equation (4) is an outgoing plane-wave representation for the reflected field in $z>z_{\max }$.

In the subdomain $z<z_{\min }$ of $D_{2}$, where $z_{\min }$ is the minimum value of $z$ on $S$, the transmitted field admits the representation

$$
\left\{P_{t}, \mathbf{V}_{t}\right\}=\iint_{-\infty}^{\infty}\left\{p_{t}, \mathbf{v}_{t}\right\} e^{i \mathbf{k}_{2}^{-} \cdot \mathbf{r}} d k_{x} d k_{y} \quad\left(z<z_{\min }\right)
$$

in which

$$
\mathbf{k}_{2}^{-}=\left(k_{x}, k_{y},-k_{z, 2}\right)
$$

where

$$
k_{z, 2}=\sqrt{\omega^{2} \rho_{2} \kappa_{2}-k_{x}^{2}-k_{y}^{2}} \quad \mathfrak{R}\left(k_{z, 2}\right), \mathcal{I}\left(k_{z, 2}\right)>0 .
$$

Equation (7) is an outgoing plane-wave representation for the transmitted field in $z<z_{\min }$.

It should be noted that the expressions (4) and (7) can in general not be continued analytically into the domain $z_{\min }<$ $z<z_{\max }$. Therefore, they can not directly be used to satisfy pointwise the boundary conditions at $S$. In the next section it is shown that the outgoing plane-wave representations are valid if we use an integrated squared error in the boundary conditions.

\section{The InTEgrated SQuared ERror Criterion}

The strategy for solving the reflection/transmission problem, is to approximate computationally the reflected field in $D_{1}$ and the transmitted field in $D_{2}$ by plane-wave representations that satisfy the corresponding source-free acoustic field equations. The plane waves are traveling in the direction of increasing $z$ in $D_{1}$ and the direction of decreasing $z$ in $D_{2}$. If approximations are denoted by adding a tilde, the plane-wave representations become

$$
\left\{\tilde{P}_{r}, \tilde{\mathbf{V}}_{r}\right\}=\iint_{-\infty}^{\infty}\left\{\tilde{p}_{r}, \tilde{\mathbf{v}}_{r}\right\} e^{i \mathbf{k}_{1}^{+} \cdot \mathbf{r}} d k_{x} d k_{y} \quad \mathbf{r} \in D_{1}
$$

and

$$
\left\{\tilde{P}_{t}, \tilde{\mathbf{V}}_{t}\right\}=\iint_{-\infty}^{\infty}\left\{\hat{p}_{t}, \tilde{\mathbf{v}}_{t}\right\} e^{i \mathbf{k}_{2}^{-} \cdot \mathbf{r}} d k_{x} d k_{y} \quad \mathbf{r} \in D_{2}
$$

where

$$
\left\{\tilde{p}_{r}, \tilde{\mathbf{v}}_{r}\right\}=i\left\{\omega \rho_{1}, \mathbf{k}_{1}^{+}\right\} \tilde{\phi}_{r}
$$

and

$$
\left\{\tilde{p}_{t}, \tilde{\mathbf{v}}_{t}\right\}=i\left\{\omega \rho_{2}, \mathbf{k}_{2}^{-}\right\} \tilde{\phi}_{t} .
$$

In view of (12), (10) satisfies (1), and in view of (13), (11) satisfies (2), provided that the right-hand sides of (10) and (11) converge in a certain sense. The scalar functions $\tilde{\phi}_{r, t}=\tilde{\phi}_{r, t}\left(k_{x}, k_{y}\right)$ are approximations of the plane-wave components $\phi_{r, t}\left(k_{x}, k_{y}\right)$ of the velocity potential.

The plane-wave components $\phi_{r, t}\left(k_{x}, k_{y}\right)$ are obtained by minimizing the error in the boundary conditions at the interface. The question is whether we can also use (10) and (11) on the interface between the two media. This question arises because the plane-wave representations can not directly be used inside the grooves of the irregular interface. According to Millar [13], the use of outgoing plane-wave representations on the interface can yield valid results if we meet two requirements. The first requirement is that we should use an integrated squared error criterion for the approximation in the boundary conditions. The second requirement is that the interface should not contain sharp edges. If we meet these two requirements and drive the error in the boundary conditions towards zero, the solution in the exterior domain should converge in the mean towards the exact solution. The use of outgoing waves is sufficient for convergence in the mean sense, although possibly at the cost of worse numerical convergence. The sufficiency of the mean-squared error minimization in the boundary conditions is shown in Appendices A, B, and C. There we show that the fields in domains $D_{1}$ and $D_{2}$ converge in the mean if we drive the integrated squared error in the boundary conditions towards zero.

In other words, if we drive the integrated squared error ERR to zero:

$$
E R R=\iint_{s}\left(\left|F_{P}\right|^{2}+\left|F_{V}\right|^{2}\right) d A \rightarrow 0
$$


where (the right arrow denotes convergence in the mean)

$$
\begin{aligned}
& F_{P}=Y_{0}^{1 / 2}\left(P_{i}+\tilde{P}_{r}-\tilde{P}_{t}\right) \\
& F_{V}=Z_{0}^{1 / 2} \boldsymbol{\nu} \cdot\left(\mathbf{V}_{i}+\tilde{\mathbf{V}}_{r}-\tilde{\mathbf{V}}_{t}\right)
\end{aligned}
$$

then we have convergence for the reflected field

$$
\left\{\tilde{P}_{r}(\mathbf{r}), \tilde{\mathbf{V}}_{r}(\mathbf{r})\right\} \rightarrow\left\{P_{r}(\mathbf{r}), \mathbf{V}_{r}(\mathbf{r})\right\} \quad \mathbf{r} \in D_{1}
$$

as well as for the transmitted field

$$
\left\{\tilde{P}_{t}(\mathbf{r}), \tilde{\mathbf{V}}_{t}(\mathbf{r})\right\} \rightarrow\left\{P_{t}(\mathbf{r}), \mathbf{V}_{t}(\mathbf{r})\right\} \quad \mathbf{r} \in D_{2} .
$$

The factor $Y_{0}=\left(\kappa_{0} / \rho_{0}\right)^{1 / 2}$ is the acoustic wave admittance in a reference medium and $Z_{0}=\left(\rho_{0} / \kappa_{0}\right)^{1 / 2}$ is the wave impedance in the same medium. These values act as normalization constants. In the next section, we shall show how, in an iterative way, we can minimize $E R R$ in (14).

\section{ITERATIVE MINIMIZATION OF THE INTEGRATED SQUARED ERROR}

The solution of the reflection/transmission problem can be found by an iterative minimization of the integrated squared error. The tilde over the different symbols to denote the approximation procedure will be omitted. We assume the existence of an iterative procedure, in which $n$ steps have been carried out. The iterative procedure has led to the values $\phi_{r}^{(n)}$ and $\phi_{t}^{(n)}$ which can be used in (12) and (13). The corresponding approximate field values are

$$
\begin{array}{ll}
\left\{P_{r}^{(n)}, \mathbf{V}_{r}^{(n)}\right\}=\iint_{-\infty}^{\infty}\left\{p_{r}^{(n)}, \mathbf{v}_{r}^{(n)}\right\} e^{i \mathbf{k}_{1}^{+} \cdot \mathbf{r}} d k_{x} d k_{y} & \mathbf{r} \in D_{1} \\
\left\{P_{t}^{(n)}, \mathbf{V}_{t}^{(n)}\right\}=\iint_{-\infty}^{\infty}\left\{p_{t}^{(n)}, \mathbf{v}_{t}^{(n)}\right\} e^{i \mathbf{k}_{2}^{-} \cdot \mathbf{r}} d k_{x} d k_{y} & \mathbf{r} \in D_{2}
\end{array}
$$

where

$$
\begin{aligned}
& \left\{p_{r}^{(n)}, \mathbf{v}_{r}^{(n)}\right\}=i\left\{\omega \rho_{1}, \mathbf{k}_{1}^{+}\right\} \phi_{r}^{(n)} \\
& \left\{p_{t}^{(n)}, \mathbf{v}_{t}^{(n)}\right\}=i\left\{\omega \rho_{2}, \mathbf{k}_{2}^{-}\right\} \phi_{t}^{(n)}
\end{aligned}
$$

the integrated squared error $E R R^{(n)}$ after $n$ steps of iteration is

$$
E R R^{(n)}=\iint_{S}\left(\left|F_{P}^{(n)}\right|^{2}+\left|F_{V}^{(n)}\right|^{2}\right) d A
$$

in which the deviations $F_{P}^{(n)}=F_{P}^{(n)}(\mathbf{r}), F_{V}^{(n)}=F_{V}^{(n)}(\mathbf{r})$ are given by

$$
\begin{aligned}
& F_{P}^{(n)}=Y_{0}^{1 / 2}\left(P_{i}+P_{r}^{(n)}-P_{t}^{(n)}\right) \\
& F_{V}^{(n)}=Z_{0}^{1 / 2} \boldsymbol{\nu} \cdot\left(\mathbf{V}_{i}+\mathbf{V}_{r}^{(n)}-\mathbf{V}_{t}^{(n)}\right)
\end{aligned}
$$

In going from the $(n-1)$ st step to the $n$ th, we take

$$
\phi_{r, t}^{(n)}=\phi_{r, t}^{(n-1)}+\eta^{(n)} g_{r, t}^{(n)}
$$

where $\eta^{(n)}$ is a variational parameter and $g_{r, t}^{(n)}=g_{r, t}^{(n)}\left(k_{x}, k_{y}\right)$ are suitably chosen directions. The construction of these directions will be discussed in Section V. Upon using (22), the deviations become

$$
F_{P, V}^{(n)}=F_{P, V}^{(n-1)}-\eta^{(n)} f_{P, V}^{(n)}
$$

in which

$$
\begin{aligned}
f_{P}^{(n)}=-i \omega \rho_{1} Y_{0}^{1 / 2} \iint_{-\infty}^{\infty} g_{r}^{(n)} e^{i \mathbf{k}_{1}^{+} \cdot \mathbf{r}} d k_{x} d k_{y} \\
+i \omega \rho_{2} Y_{0}^{1 / 2} \iint_{-\infty}^{\infty} g_{t}^{(n)} e^{i \mathbf{k}_{2}^{-} \cdot \mathbf{r}} d k_{x} d k_{y} \\
f_{V}^{(n)}=-i Z_{0}^{1 / 2} \iint_{-\infty}^{\infty} \boldsymbol{\nu} \cdot \mathbf{k}_{1}^{+} g_{r}^{(n)} e^{i \mathbf{k}_{1}^{+} \cdot \mathbf{r}} d k_{x} d k_{y} \\
+i Z_{0}^{1 / 2} \iint_{-\infty}^{\infty} \nu \cdot \mathbf{k}_{2}^{-} g_{t}^{(n)} e^{i \mathbf{k}_{2}^{-} \cdot \mathbf{r}} d k_{x} d k_{y} .
\end{aligned}
$$

The expression of $E R R^{(n)}$ can be written as

$$
\begin{aligned}
E R R^{(n)}= & E R R^{(n-1)}-2 \Re\left(\eta^{(n)} A^{(n)}\right)+\left|\eta^{(n)}\right|^{2} B^{(n)} \\
= & E R R^{(n-1)}-\left|A^{(n)}\right|^{2} / B^{(n)} \\
& +\left|\eta^{(n)}-A^{(n) *} / B^{(n)}\right|^{2} B^{(n)}
\end{aligned}
$$

in which

$$
A^{(n)}=\iint_{S}\left(F_{P}^{(n-1) *} f_{P}^{(n)}+F_{V}^{(n-1) *} f_{V}^{(n)}\right) d A
$$

and

$$
B^{(n)}=\iint_{S}\left(\left|f_{P}^{(n)}\right|^{2}+\left|f_{V}^{(n)}\right|^{2}\right) d A
$$

where the asterisk denotes the complex conjugate. The righthand side of (26) has, as a function of $\eta^{(n)}$, a minimum at

$$
\eta^{(n)}=A^{(n) *} / B^{(n)} .
$$

Substituting this value of $\eta^{(n)}$ in (26)

$$
E R R^{(n)}=E R R^{(n-1)}-\left|A^{(n)}\right|^{2} / B^{(n)}
$$

from which it follows that $E R R^{(n)}<E R R^{(n-1)}$, provided $A^{(n)} \neq 0$. This latter condition puts some restriction on the choice of the variational functions $g_{r, t}^{(n)}$. If $A^{(n)} \neq 0$, an improvement in the satisfaction of the boundary conditions is arrived at, although it is in a mean sense.

Substitution of (29) in (23) leads to

$$
F_{P, V}^{(n)}=F_{P, V}^{(n-1)}-\left(A^{(n) *} / B^{(n)}\right) f_{P, V}^{(n)} .
$$

From this, it follows that

$$
\iint_{S}\left(F_{P}^{(n) *} f_{P}^{(n)}+F_{V}^{(n) *} f_{V}^{(n)}\right) d A=0 .
$$

This orthogonality property on $S$ will be used later. For the definition of the search directions in the next section we also want to make the dependence of $A^{(n)}$ on $g_{r, t}^{(n)}$ explicit. This can be accomplished by interchanging the integrations in (27) and by writing

$$
A^{(n)}=\iint_{-\infty}^{\infty}\left(s_{r}^{(n-1) *} g_{r}^{(n)}+s_{t}^{(n-1) *} g_{t}^{(n)}\right) d k_{x} d k_{y}
$$


where

$$
\begin{aligned}
& s_{r}^{(n) *}=-i \omega \rho_{1} Y_{0}^{1 / 2} \iint_{S} F_{P}^{(n) *} e^{i \mathbf{k}_{1}^{+} \cdot \mathbf{r}} d A \\
&-i Z_{0}^{1 / 2} \iint_{S} \boldsymbol{\nu} \cdot \mathbf{k}_{1}^{+} F_{V}^{(n) *} e^{i \mathbf{k}_{1}^{+} \cdot \mathbf{r}} d A \\
& s_{t}^{(n) *}=+i \omega \rho_{2} Y_{0}^{1 / 2} \iint_{S} F_{P}^{(n) *} e^{i \mathbf{k}_{2}^{-} \cdot \mathbf{r}} d A \\
&+i Z_{0}^{1 / 2} \iint_{S} \boldsymbol{\nu} \cdot \mathbf{k}_{2}^{-} F_{V}^{(n) *} e^{i \mathbf{k}_{2}^{-} \cdot \mathbf{r}} d A .
\end{aligned}
$$

The symbols $s_{r}^{(n)}$ and $s_{t}^{(n)}$ denote the steepest-descent directions for the reflected and transmitted fields, respectively.

With this, substitution of (24) and (25) in (32) leads to

$$
\iint_{-\infty}^{\infty}\left(s_{r}^{(n) *} g_{r}^{(n)}+s_{t}^{(n) *} g_{t}^{(n)}\right) d k_{x} d k_{y}=0
$$

showing that $\left\{s_{r}^{(n)}, s_{t}^{(n)}\right\}$ is orthogonal to $\left\{g_{r}^{(n)}, g_{t}^{(n)}\right\}$ in the spectral domain.

In the next section we discuss a particular choice of $g_{r, t}^{(n)}$, which up to now has been completely arbitrary.

\section{The Conjugate Gradient Directions}

As follows from Section IV, an iterative improvement in the satisfaction of the boundary conditions, at the interface is only achieved if, in each iteration, $A^{(n)} \neq 0$. This condition is fulfilled when we take the conjugate gradient directions. The conjugate gradient directions for solving a discrete system of linear equations can be found in [15], [16]. In the presen case however, we use a continuous form of the conjugate gradient method [10], [17]. The continuous conjugate gradient directions are given by

$$
g_{r, t}^{(n)}=s_{r, t}^{(n-1)}+\left(A^{(n)} / A^{(n-1)}\right) g_{r, t}^{(n-1)} \quad n \geq 2
$$

while

$$
g_{r, t}^{(1)}=s_{r, t}^{(0)}
$$

and where $s_{r, t}^{(n)}$ are the steepest-descent directions. These steepest-descent directions are orthogonal in the $k_{x}, k_{y}$-space [10]. However, the directions $g_{r, t}^{(n)}$ are not orthogonal in the $k_{x}, k_{y}$-space, but the functions $f_{P, V}^{(n)}$ are orthogonal in the conjugate $x, y$-space. Therefore we call the directions $g_{r, t}^{(n)}$ the conjugate gradient directions.

Substituting these expressions in the spectral domain equivalent of (27), viz. (33), and using the orthogonality relation (36) we obtain

$$
A^{(n)}=\iint_{-\infty}^{\infty}\left(\left|s_{r}^{(n-1)}\right|^{2}+\left|s_{t}^{(n-1)}\right|^{2}\right) d k_{x} d k_{y}
$$

which shows that $A^{(n)}$ is real and positive, unless $s_{r, t}^{(n-1)}$ vanishes. However, in the latter case, we have arrived at the exact solution in the iteration $n-1$.

At this point, the iteration scheme is fully defined. The complete iteration scheme is shown in Appendix D.

\section{NUMERICAL RESULTS}

\section{A. General}

All the integrations of the algorithm in Appendix D were calculated as simple summations of the discrete function values. The convergence properties of the algorithm depend strongly on the maximum value of the interface slope. In general, the larger the value for the maximum slope, the slower the convergence. The convergence properties for sinusoidal interfaces can be compared to those of the modified Rayleigh method discussed in [7]. The convergence is virtually independent of the period of the sinusoidal interface, although the spatial integrations are less accurate for sinusoidal interfaces with large periods.

After a certain number of iteration steps the integrated squared error decreases very slowly and the error asymptotically reaches a steady value. The speed of convergence does not depend on the contrast in the medium parameters, although the final error is lower if the contrast is lower. Experiments with the step size showed that the calculations gave consistent results from a spatial integration step size $\Delta x \leq 0.2 \lambda$, where $\lambda$ is the smallest wavelength in both media.

For the numerical implementation described in this paper, the convergence becomes worse if evanescent waves are included. Evanescent waves would be required for approximating fields with arbitrarily small error. It should be possible to include evanescent waves in combination with more accurate integrations. In our opinion, however, the resulting decrease of the error is not worth the additional computational effort. Note that the error in the boundary conditions is readily available in our iteration scheme. In this respect the error we make due to the absence of evanescent waves is checked in the iteration scheme, which is not the case in other known approximate methods. It is possible that the use of incoming waves in the grooves, in addition to outgoing waves, can improve the numerical convergence. However, the use of incoming waves is not necessary for the completeness of the solution.

\section{B. Comparison with Other Methods}

The plane-wave coefficients $\phi_{r, t}$ were also calculated by minimization of the integrated squared error in the boundary conditions using direct matrix inversion. The matrix inversion method has the disadvantage that the calculation of the matrix elements is relatively expensive. Typical cylindrical interfaces analyzed with the matrix inversion method required total computation times that were some orders of magnitude longer than with the iterative method.

In [18], the method described in this paper was compared with a rigorous integral equation method. The latter method was based on surface integral equations and free space Green's functions, where the solution was obtained iteratively or by direct matrix inversion. From the results presented there it could be seen that the numerical efficiency of the present conjugate gradient plane-wave method is high compared with the numerical efficiency of the conjugate gradient integral equation method. However, if accurate results are required in 


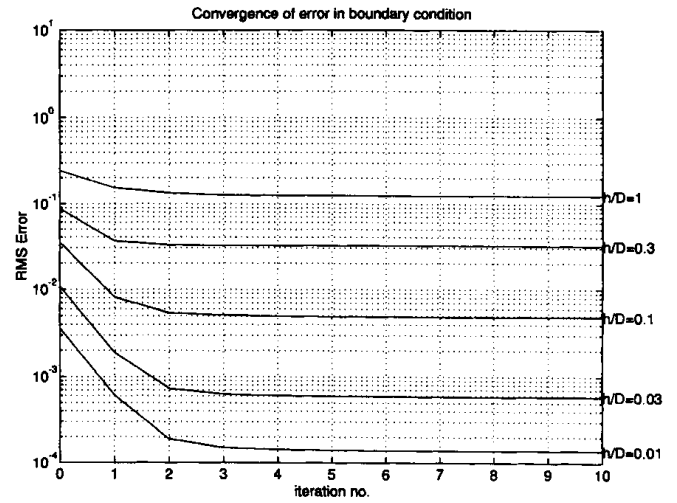

Fig. 2. Convergence properties for plane-wave incidence and a sinusoidal interface with a period of $D=2.3 \lambda$.

the case of high contrast between the media and/or very rough surfaces, then the integral equation method may be necessary.

\section{Examples}

I. Convergence for a Sinusoidal Interface: The iterative scheme of Appendix D was applied to the lossless 2D-case for an interface with a sinusoidal profile $z=0.5 h \sin (2 \pi x / D)$, where $D$ is the length of one period of the interface. The incident wave was a plane wave at normal incidence. The convergence properties of the algorithm are shown in Fig. 2 , with $h / D$ as parameter. The quantity shown in $\widehat{E R R}^{(n)}$, which is the root-mean-square error normalized to the case of vanishing reflected and transmitted fields, i.e.

$$
\widehat{E R R}^{(n)}=\left(\frac{E R R^{(n)}}{\iint_{S}\left(Y_{0}\left|P_{i}\right|^{2}+Z_{0}\left|\boldsymbol{\nu} \cdot \mathbf{V}_{i}\right|^{2}\right) d A}\right)^{1 / 2}
$$

The spatial integrations over surface $A$ where replaced by integrations over the path length $l$ along the surface. The number of plane waves, i.e., the number of discrete $k_{x}$ values, was 512 . The maximum value of $\left|k_{x}\right|$ was chosen to be the largest value for which all the plane waves in both media are nonevanescent, i.e., $\max \left\{\left|k_{x}\right|\right\}=\min \left\{k_{1}, k_{2}\right\}$. The medium parameters were chosen to be $\rho_{2} / \rho_{1}=1$ and $\kappa_{2} / \kappa_{1}=1.108$, which is equivalent to a sound speed contrast of 5 percent. The number of points on the surface $S$ was 512 with a spacing of $\Delta x=0.2 \lambda$, with $\lambda=2 \pi / \omega \sqrt{\rho_{2} \kappa_{2}}$, the wavelength in medium 2 . The values for the reference admittance $Y_{0}$ and the reference impedance $Z_{0}$ were the geometric means of the admittances and impedances of both media. The results of the convergence for $D=2.3 \lambda$ are shown in Fig. 2 for various values of $h / D$. In Figure 3 the results are shown for $D=25.5 \lambda$. It can be seen from Figs. 2 and 3 that the convergence is better for lower values of $h / D$. A stable error value is obtained approximately after 3 to 10 iterations. The calculation time for these configurations, programmed in Matlab on a $40 \mathrm{MHz}$ Sun ELC workstation, is about 2 minutes for each iteration.

2. Beam Distortion of an Ultrasonic Array Transducer: This subsection shows the influence of an irregular interface on the beam profile of an ultrasonic array transducer. The array is

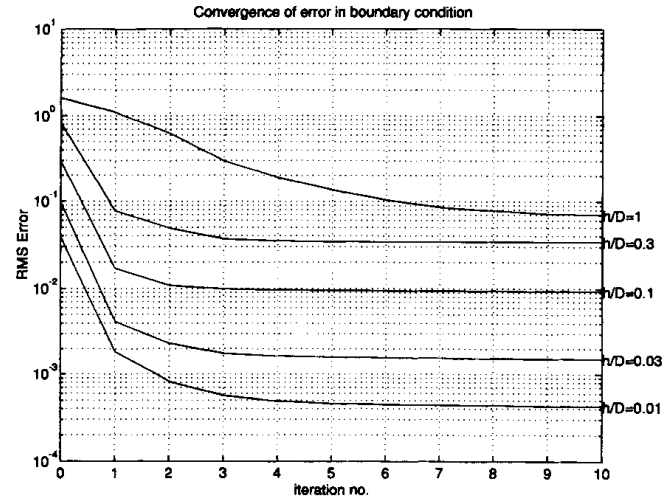

Fig. 3. Convergence properties for plane-wave incidence and a sinusoidal interface with a period of $D=25.5 \lambda$.

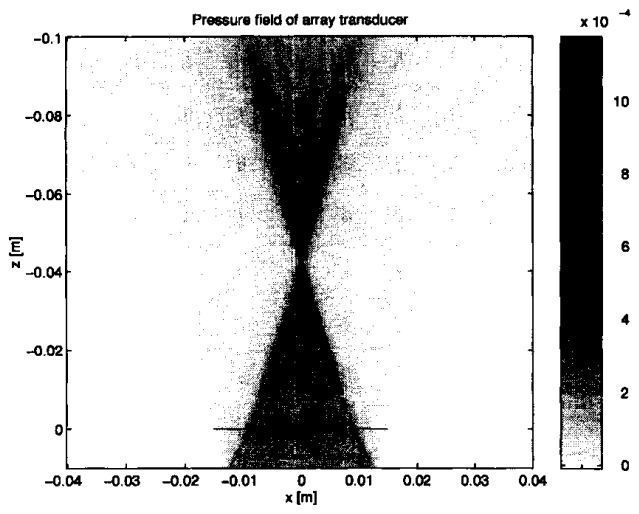

Fig. 4. Beam profile of array transducer for a plane interface separating two media. The array transducer is positioned at $z=0.01 \mathrm{~m}$ and radiates into a medium with parameters $c_{1}=1550 \mathrm{~m} / \mathrm{s}, \rho_{1}=10^{3} \mathrm{~kg} / \mathrm{m}^{3}$ at a frequency of $5 \mathrm{MHz}$. The parameters of the second medium are: $c_{2}=1473 \mathrm{~m} / \mathrm{s}, \rho_{2}=10^{3}$ $\mathrm{kg} / \mathrm{m}^{3}$. The focus is at $z=-0.04 \mathrm{~m}$.

positioned at a depth $z=10 \mathrm{~mm}$ and the mean of the interface is at $z=0$. The array radiates into a medium consisting of two layers. The layer distant from the transducer has a sound speed which is 5 percent lower than the sound speed in the medium close to the transducer. The transducer consists of 128 radiating elements, each having a width of $0.15 \mathrm{~mm}$, positioned at a grid distance of $0.2 \mathrm{~mm}$. The electronic focus is at $z=-40 \mathrm{~mm}$ (that is, at a distance of $50 \mathrm{~mm}$ from the array), in a medium with the parameters $\rho_{1}$ and $\kappa_{1}$. The array elements radiate with equal magnitudes of normal surface velocity. The frequency is $5 \mathrm{MHz}$ and the sound speeds are: $c_{1}=1550$ $\mathrm{m} / \mathrm{s}$ and $c_{2}=1473 \mathrm{~m} / \mathrm{s}$. The densities are $\rho_{1}=\rho_{2}=10^{3}$ $\mathrm{kg} / \mathrm{m}^{3}$. The other simulation parameters are described in the preceding subsection.

In Fig. 4, the beam profile obtained for a perfectly flat interface is shown, whereas in Fig. 5 the beam profile for an irregular interface is shown.

The irregular interface has a sinusoidal profile windowed by a Hamming function, with a peak-to-peak height of $h=2.3$ $\mathrm{mm}$, i.e., about 8 wavelengths. The period of the interface irregularity is $D=7.7 \mathrm{~mm}$, i.e., about 25.5 wavelengths. The 


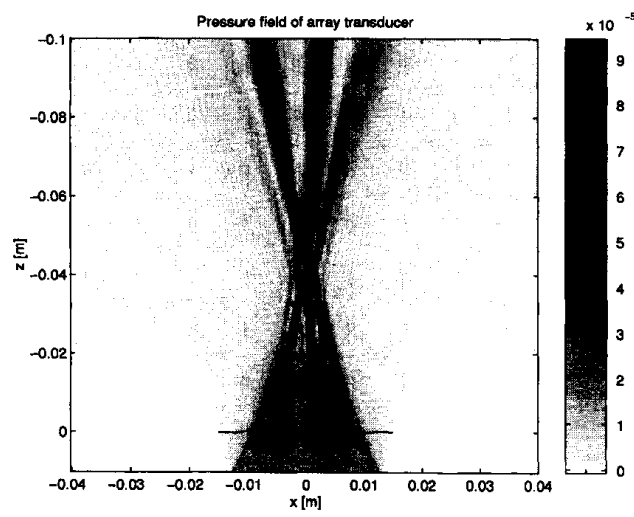

Fig. 5. Beam profile of array transducer for an irregular interface separating two media with parameters as in Fig. 1, initial guess: plane interface starting values no. of iterations: $n=10$, resulting error: $\left(E R R^{(n)} / E R R^{(0)}\right)^{1 / 2}=0.018$.

geometry of the interface is shown as a dark line in Figs. 4 and 5. The degradation of the focus due to the irregular interface is clear.

\section{CONCLUDING REMARKS}

A scheme has been developed by which the acoustic field, that is reflected and transmitted at a rough interface between two different media, can be computed in an iterative way. The method is based on a wave-function expansion technique, where the acoustic field equations are satisfied analytically, while the boundary conditions at the interface are satisfied numerically. The integrated squared error in the boundary conditions is minimized with an iterative conjugate gradient technique. The convergence of the iterative scheme is proved. The numerical implementation shows that the algorithm converges rapidly, but that there is a lower bound on the final error which can be achieved. If a sinusoidal interface is employed, lower values of height-to-period-ratio result in better convergence and lower final errors. The numerical implementation of the integrals was based on zero-order integration formulas, that is, simple summations of the integrands at discrete positions. The accuracy of the integrations can be improved by assuming a polynomial description for the interface and using analytical calculations for the resulting subintegrals.

\section{APPENDIX A}

\section{RECIPROCITY RELATION}

Two nonidentical, admissible acoustic states "A" and " $B$ " of the same angular frequency $\omega$, that are present in the same bounded domain $D$ (Fig. 1) are related via the frequencydomain reciprocity relation.

The local form of the reciprocity relation can be obtained by using the acoustic equations with source distributions $Q$ and $\mathbf{F}$

$$
\begin{aligned}
\nabla \cdot \mathbf{V}-i \omega \kappa P & =Q \\
\nabla P-i \omega \rho \mathbf{V} & =\mathbf{F}
\end{aligned}
$$
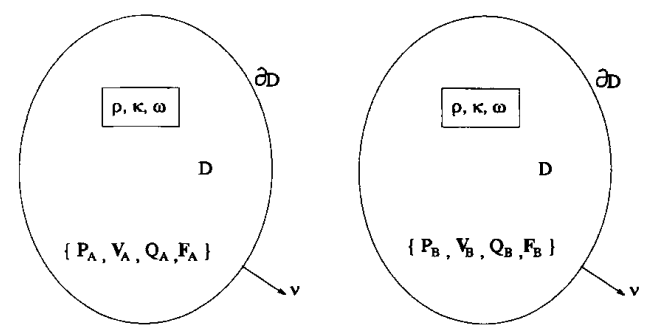

Fig. 6. States "A" and "B" in the bounded domain $D$.

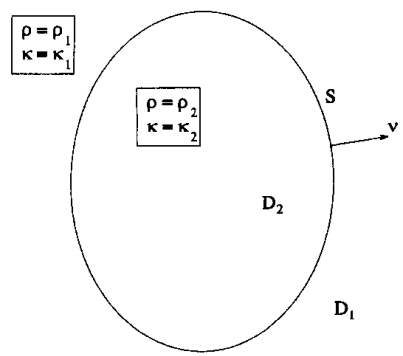

Fig. 7. The configuration of the two-media problem.

which results in [12]

$$
\begin{aligned}
& \nabla \cdot\left(P_{A} \mathbf{V}_{B}-P_{B} \mathbf{V}_{A}\right) \\
& \quad=\mathbf{F}_{A} \cdot \mathbf{V}_{B}+Q_{B} P_{A}-\mathbf{F}_{B} \cdot \mathbf{V}_{A}-Q_{A} P_{B} .
\end{aligned}
$$

Integration of the local reciprocity relation (A.2) over $D$ and application of Gauss' divergence theorem yield the global reciprocity relation

$$
\begin{aligned}
& \iint_{\partial D} \boldsymbol{\nu} \cdot\left(P_{A} \mathbf{V}_{B}-P_{B} \mathbf{V}_{A}\right) d A \\
&=\iiint_{D}\left(\mathbf{F}_{A} \cdot \mathbf{V}_{B}+Q_{B} P_{A}-\mathbf{F}_{B} \cdot \mathbf{V}_{A}-Q_{A} P_{B}\right) d V
\end{aligned}
$$

In (A.3), $\partial D$ denotes the boundary surface of $D$ and $\nu$ denotes the unit vector in the direction of the outward normal to $\partial D$.

\section{APPENDIX B}

\section{GREen's STATE of THE Two-Media Problem}

Surface source-integral representations for the reflected and transmitted fields in the two-media problem of Fig. 7 can be derived by using the reciprocity relation of Appendix A.

It is assumed that sources located in $D_{1}$ excite an incident field $\left\{P_{i}, \mathbf{V}_{i}\right\}$. The reflected and transmitted field satisfy the homogeneous acoustic equations in $D_{1}$ and $D_{2}$

$$
\begin{array}{r}
\nabla \cdot \mathbf{V}-i \omega \kappa P=0 \\
\nabla P-i \omega \rho \mathbf{V}=\mathbf{0}
\end{array}
$$

where in $D_{1}: \rho=\rho_{1}, \kappa=\kappa_{1}$ and $D_{2}: \rho=\rho_{2}, \kappa=\kappa_{2}$. The reflected field, in $D_{1}$, is denoted by $\{P, \mathbf{V}\}=\left\{P_{r}, \mathbf{V}_{r}\right\}$. The transmitted field, in $D_{2}$, is denoted by $\{P, \mathbf{V}\}=\left\{P_{t}, \mathbf{V}_{t}\right\}$. The reflected field satisfies the radiation condition at infinity. 
We define, in $R^{3}$, the volume injection Green's state as the field $\left\{P_{q}, \mathbf{V}_{q}\right\}$ that satisfies the conditions [12]

$$
\begin{aligned}
\nabla \cdot \mathbf{V}_{q}-i \omega \kappa P_{q} & =q \delta\left(\mathbf{r}-\mathbf{r}^{\prime}\right) \\
\nabla P_{q}-i \omega \rho \mathbf{V}_{q} & =\mathbf{0} \quad \mathbf{r} \in D_{1} \cup D_{2}, \quad r^{\prime} \in R^{3}(
\end{aligned}
$$

where $\rho=\rho_{1}, \kappa=\kappa_{1}$ in $D_{1}$ and in $\rho=\rho_{2}, \kappa=\kappa_{2}$ in $D_{2}$, while

$$
\begin{aligned}
P_{q} & =\text { continuous across } S \\
\nu \cdot \mathrm{V}_{q} & =\text { continuous across } S
\end{aligned}
$$

and in which $\boldsymbol{\nu}$ is the unit vector along the outward normal to $S$. The field $\left\{P_{q}, \mathbf{V}_{q}\right\}$ satisfies the radiation condition at infinity. Application of (A.3) to the domain $D_{1}$ and to the fields $\left\{P_{A}, \mathbf{V}_{A}\right\}=\left\{P_{r}, \mathbf{V}_{r}\right\}$ and $\left\{P_{B}, \mathbf{V}_{B}\right\}=\left\{P_{q}, \mathbf{V}_{q}\right\}$ yields

$\iint_{S} \boldsymbol{\nu} \cdot\left(P_{r} \mathbf{V}_{q}-P_{q} \mathbf{V}_{r}\right) d A=\left\{q P_{r}\left(\mathbf{r}^{\prime}\right), 0\right\} \quad \mathbf{r}^{\prime} \in\left\{D_{1}, D_{2}\right\}$

Application of (A.3) to the domain $D_{2}$ and to the fields $\left\{P_{A}, \mathbf{V}_{A}\right\}=\left\{P_{t}, \mathbf{V}_{t}\right\}$ and $\left\{P_{B}, \mathbf{V}_{B}\right\}=\left\{P_{q}, \mathbf{V}_{q}\right\}$ yields

$$
\begin{aligned}
\iint_{S} \boldsymbol{\nu} \cdot\left(P_{t} \mathbf{V}_{q}-P_{q} \mathbf{V}_{t}\right) d A=\left\{0,-q P_{t}\left(\mathbf{r}^{\prime}\right)\right\} \\
\mathbf{r}^{\prime} \in\left\{D_{1}, D_{2}\right\} .
\end{aligned}
$$

Subtraction of (B.5) from (B.4), and use of (B.3) leads to the expression

$$
\begin{aligned}
\iint_{S} \boldsymbol{\nu} \cdot\left[\left(P_{r}\right.\right. & \left.\left.-P_{t}\right) \mathbf{V}_{q}-\left(\mathbf{V}_{r}-\mathbf{V}_{t}\right) P_{q}\right] d A \\
& =\left\{q P_{r}\left(\mathbf{r}^{\prime}\right), q P_{t}\left(\mathbf{r}^{\prime}\right)\right\} \quad \mathbf{r}^{\prime} \in\left\{D_{1}, D_{2}\right\} .
\end{aligned}
$$

The field $\left\{P_{q}, \mathbf{V}_{q}\right\}$ is just the acoustic field excited by a point source with volume injection $Q=q \delta\left(\mathbf{r}-\mathbf{r}^{\prime}\right)$ and depends linearly on $q$.

Similarly, when the volume force Green's state $\left\{P_{f}, \mathbf{V}_{f}\right\}$ that is generated by the source distribution

$$
\mathbf{F}=\mathbf{f} \delta\left(\mathbf{r}-\mathbf{r}^{\prime}\right)
$$

is introduced, we arrive at the expression

$$
\begin{aligned}
& \iint_{S} \boldsymbol{\nu} \cdot {\left[\left(P_{r}-P_{t}\right) \mathbf{V}_{f}-\left(\mathbf{V}_{r}-\mathbf{V}_{t}\right) P_{f}\right] d A } \\
&=-\left\{\mathbf{f} \cdot \mathbf{V}_{r}\left(\mathbf{r}^{\prime}\right), \mathbf{f} \cdot \mathbf{V}_{t}\left(\mathbf{r}^{\prime}\right)\right\} \quad \mathbf{r}^{\prime} \in\left\{D_{1}, D_{2}\right\} .
\end{aligned}
$$

The field $\left\{P_{f}, \mathbf{V}_{f}\right\}$ depends linearly on $\mathbf{f}$.

Since

$$
\begin{aligned}
P_{i}+P_{r}-P_{t} & =0 \\
\left(\mathbf{V}_{i}+\mathbf{V}_{r}-\mathbf{V}_{t}\right) & =0
\end{aligned} \quad \mathbf{r} \in S
$$

we arrive at the result

$$
\begin{array}{r}
-\left\{q P_{r}\left(\mathbf{r}^{\prime}\right), q P_{t}\left(\mathbf{r}^{\prime}\right)\right\}=\iint_{S} \boldsymbol{\nu} \cdot\left(P_{i} \mathbf{V}_{q}-\mathbf{V}_{i} P_{q}\right) d A \\
\mathbf{r}^{\prime} \in\left\{D_{1}, D_{2}\right\}
\end{array}
$$

$$
\begin{array}{r}
\left\{\mathbf{f} \cdot \mathbf{V}_{r}\left(\mathbf{r}^{\prime}\right), \mathbf{f} \cdot \mathbf{V}_{t}\left(\mathbf{r}^{\prime}\right)\right\}=\iint_{S} \boldsymbol{\nu} \cdot\left(P_{i} \mathbf{V}_{f}-\mathbf{V}_{i} P_{f}\right) d A \\
\mathbf{r}^{\prime} \in\left\{D_{1}, D_{2}\right\} .
\end{array}
$$

These are the desired surface-source representations for the reflected and the transmitted fields. If $\left\{P_{q}, V_{q}\right\}$ were known, (B.10) would lead to $P_{r, t}$ at any $\mathbf{r}^{\prime} \in D_{1,2}$, while if $\left\{P_{f}, \mathbf{V}_{f}\right\}$ were known, (B.11) would lead to $\mathbf{V}_{r, t}$ at any $\mathbf{r}^{\prime} \in D_{1,2}$.

APPENDIX C

\section{SUFFICIENCY OF AN ERROR CRITERION} IN THE BOUNDARY CONDITIONS

In this appendix it is shown that a certain approximation in the boundary conditions leads to a certain degree of approximation in the reflected and transmitted fields. The existence is assumed of some field $\left\{\tilde{P}_{r}, \tilde{\mathbf{V}}_{r}\right\}$ in $D_{1}$ and some field $\left\{\tilde{P}_{t}, \tilde{\mathbf{V}}_{t}\right\}$ in $D_{2}$, which satisfy the acoustic equations (B.1) and the radiation condition at infinity. It is also assumed that they violate the boundary conditions at $S$, i.e.,

$$
\begin{gathered}
P_{i}+\tilde{P}_{r} \neq \tilde{P}_{t} \\
\boldsymbol{\nu} \cdot \mathbf{V}_{i}+\boldsymbol{\nu} \cdot \tilde{\mathbf{V}}_{r} \neq \boldsymbol{\nu} \cdot \tilde{\mathbf{V}}_{t} \quad \mathbf{r} \in S
\end{gathered}
$$

Since the acoustic equations and the radiation condition are satisfied, relations of the type (B.6) and (B.8) also hold for $\left\{\tilde{P}_{r}, \tilde{\mathbf{V}}_{r}\right\}$ in $D_{1}$ and $\left\{\tilde{P}_{t}, \tilde{\mathbf{V}}_{t}\right\}$ in $D_{2}$

$$
\begin{aligned}
& \left\{q \tilde{P}\left(\mathbf{r}^{\prime}\right), q \tilde{P}_{t}\left(\mathbf{r}^{\prime}\right)\right\} \\
& =\iint_{S} \boldsymbol{\nu} \cdot\left[\left(\tilde{P}_{r}-\tilde{P}_{t}\right) \mathbf{V}_{q}-\left(\tilde{\mathbf{V}}_{r}-\tilde{\mathbf{V}}_{t}\right) P_{q}\right] d A \\
& \qquad \mathbf{r}^{\prime} \in\left\{D_{1}, D_{2}\right\} \\
& -\left\{\mathbf{f} \cdot \tilde{\mathbf{V}}_{r}\left(\mathbf{r}^{\prime}\right), \mathbf{f} \cdot \tilde{\mathbf{V}}_{t}\left(\mathbf{r}^{\prime}\right)\right\} \\
& =\iint_{S} \boldsymbol{\nu} \cdot\left[\left(\tilde{P}_{r}-\tilde{P}_{t}\right) \mathbf{V}_{f}-\left(\tilde{\mathbf{V}}_{r}-\tilde{\mathbf{V}}_{t}\right) P_{f}\right] d A \\
& \quad \mathbf{r}^{\prime} \in\left\{D_{1}, D_{2}\right\} .
\end{aligned}
$$

Combining (C.2) with (B.10), and (C.3) with (B.11), and applying Cauchy-Schwarz's inequality, we obtain the following inequalities

$$
\begin{gathered}
\left|q P_{r}\left(\mathbf{r}^{\prime}\right)-q \tilde{P}_{r}\left(\mathbf{r}^{\prime}\right)\right|^{2} \\
\leq\left(E R R_{P}+E R R_{V}\right) \iint_{S}\left(Z_{0}\left|\boldsymbol{\nu} \cdot \mathbf{V}_{q}\right|^{2}+Y_{0}\left|P_{q}\right|^{2}\right) d A \\
\mathbf{r}^{\prime} \in D_{1} \\
\left|f \cdot \mathbf{V}_{r}\left(\mathbf{r}^{\prime}\right)-f \cdot \tilde{\mathbf{V}}_{r}\left(\mathbf{r}^{\prime}\right)\right|^{2} \\
\leq\left(E R R_{P}+E R R_{V}\right) \iint_{S}\left(Z_{0}\left|\boldsymbol{\nu} \cdot \mathbf{V}_{f}\right|^{2}+Y_{0}\left|P_{f}\right|^{2}\right) d A \\
\mathbf{r}^{\prime} \in D_{1}
\end{gathered}
$$

$$
\begin{aligned}
& \left|q P_{t}\left(\mathbf{r}^{\prime}\right)-q \tilde{P}_{t}\left(\mathbf{r}^{\prime}\right)\right|^{2} \\
& \leq\left(E R R_{P}+E R R_{V}\right) \iint_{S}\left(Z_{0}\left|\nu \cdot \mathbf{V}_{q}\right|^{2}+Y_{0}\left|P_{q}\right|^{2}\right) d A \\
& \quad \mathbf{r}^{\prime} \in D_{2}
\end{aligned}
$$




$$
\begin{aligned}
\left|\mathbf{f} \cdot \mathbf{V}_{t}\left(\mathbf{r}^{\prime}\right)-\mathbf{f} \cdot \tilde{\mathbf{V}}_{t}\left(\mathbf{r}^{\prime}\right)\right|^{2} \\
\leq\left(E R R_{P}+E R R_{V}\right) \iint_{S}\left(Z_{0}\left|\boldsymbol{\nu} \cdot \mathbf{V}_{f}\right|^{2}+Y_{0}\left|P_{f}\right|^{2}\right) d A \\
\quad \mathbf{r}^{\prime} \in D_{2}
\end{aligned}
$$

where the integrated squared errors in the pressure field and the particle velocity field at $S$ have been introduced as

$$
\begin{array}{r}
E R R_{P}=\iint_{S} Y_{0}\left|P_{i}+\tilde{P}_{r}-\tilde{P}_{t}\right|^{2} d A \\
E R R_{V}=\iint_{S} Z_{0}\left|\boldsymbol{\nu} \cdot\left(\mathbf{V}_{i}+\tilde{\mathbf{V}}_{r}-\tilde{\mathbf{V}}_{t}\right)\right|^{2} d A .
\end{array}
$$

The factor $Y_{0}=\left(\kappa_{0} / \rho_{0}\right)^{1 / 2}$ is the wave admittance in a reference medium, while $Z_{0}=\left(\rho_{0} / \kappa_{0}\right)^{1 / 2}$ is the wave impedance in the same reference medium. They have been introduced for dimensional convenience and the values are not critical. From the foregoing results it can be concluded that, if

$$
\left(E R R_{P}+E R R_{V}\right) \rightarrow 0
$$

then

$$
\begin{aligned}
q \tilde{P}_{r}\left(\mathbf{r}^{\prime}\right) & \rightarrow q P_{r}\left(\mathbf{r}^{\prime}\right) \\
\mathbf{f} \cdot \tilde{\mathbf{V}}_{r}\left(\mathbf{r}^{\prime}\right) & \rightarrow \mathbf{f} \cdot \mathbf{V}_{r}\left(\mathbf{r}^{\prime}\right)
\end{aligned} \quad \mathbf{r}^{\prime} \in D_{1}
$$

and

$$
\begin{aligned}
q \tilde{P}_{t}\left(\mathbf{r}^{\prime}\right) \rightarrow q P_{t}\left(\mathbf{r}^{\prime}\right) \\
\mathbf{f} \cdot \tilde{\mathbf{V}}_{t}\left(\mathbf{r}^{\prime}\right) \rightarrow \mathbf{f} \cdot \mathbf{V}_{t}\left(\mathbf{r}^{\prime}\right)
\end{aligned} \quad \mathbf{r}^{\prime} \in D_{2}
$$

because the surface integrals in (C.4-C.7) containing the Green's states are bounded $\left(\mathbf{r}^{\prime} \ni S\right)$. The arbitrariness of $q$ and $\mathbf{f}$ in (C.11) and (C.12) leads to

$$
\begin{array}{ll}
\left\{\tilde{P}_{r}, \tilde{\mathbf{V}}_{r}\right\} \rightarrow\left\{P_{r}, \mathbf{V}_{r}\right\} & \mathbf{r}^{\prime} \in D_{1} \\
\left\{\tilde{P}_{t}, \tilde{\mathbf{V}}_{t}\right\} \rightarrow\left\{P_{t}, \mathbf{V}_{t}\right\} & \mathbf{r}^{\prime} \in D_{2}
\end{array}
$$

The derivation of the iterative scheme in the main text is based on the error criterion (C.10).

\section{APPENDIX D}

\section{ITERATIVE SCHEME}

The iterative scheme has to be started with the estimates $\phi_{r, t}^{(0)}$. The solutions for a plane interface [14] can be used as starting values. If the incident velocity potential $\Phi_{i}$ at the plane interface $z=0$ is known, the incident field $\left\{P_{i}, \mathbf{V}_{i}\right\}$ at the rough surface $S$ and the estimates $\phi_{r, t}^{(0)}$ can be calculated.

Plane interface starting values

$$
\begin{gathered}
\phi_{i}=\frac{1}{4 \pi^{2}} \iint_{-\infty}^{\infty} \boldsymbol{\Phi}_{i}(x, y, z=0) e^{-i\left(k_{x} x+k_{y} y\right)} d x d y \\
P_{i}=i \omega \rho_{1} \iint_{-\infty}^{\infty} \phi_{i} e^{i \mathbf{k}_{1}^{-} \cdot \mathbf{r}} d k_{x} d k_{y} \\
\mathbf{V}_{i}=i \iint_{-\infty}^{\infty} \mathbf{k}_{1}^{-} \phi_{i} e^{i \mathbf{k}_{1}^{-} \cdot \mathbf{r}} d k_{x} d k_{y}
\end{gathered}
$$

$$
\phi_{r}^{(0)}=\frac{\rho_{2} k_{z, 1}-\rho_{1} k_{z, 2}}{\rho_{2} k_{z, 1}+\rho_{1} k_{z, 2}} \phi_{i}, \quad \phi_{t}^{(0)}=\frac{2 \rho_{1} k_{z, 1}}{\rho_{2} k_{z, 1}+\rho_{1} k_{z, 2}} \phi_{i}
$$

Initialization

$$
\begin{gathered}
P_{r}^{(0)}=i \omega \rho_{1} \iint_{-\infty}^{\infty} \phi_{r}^{(0)} e^{i \mathbf{k}_{1}^{+} \cdot \mathbf{r}} d k_{x} d k_{y} \\
\mathbf{V}_{r}^{(0)}=i \iint_{-\infty}^{\infty} \mathbf{k}_{1}^{+} \phi_{r}^{(0)} e^{i \mathbf{k}_{1}^{+} \cdot \mathbf{r}} d k_{x} d k_{y} \\
P_{t}^{(0)}=i \omega \rho_{2} \iint_{-\infty}^{\infty} \phi_{t}^{(0)} e^{i \mathbf{k}_{2}^{-} \cdot \mathbf{r}} d k_{x} d k_{y} \\
\mathbf{V}_{t}^{(0)}=i \iint_{-\infty}^{\infty} \mathbf{k}_{2}^{-} \phi_{t}^{(0)} e^{i k_{2}^{-} \cdot \mathbf{r}} d k_{x} d k_{y} \\
F_{P}^{(0)}=Y_{0}^{1 / 2}\left(P_{i}+P_{r}^{(0)}-P_{t}^{(0)}\right) \\
F_{V}^{(0)}=Z_{0}^{1 / 2} \boldsymbol{\nu} \cdot\left(\mathbf{V}_{i}+\mathbf{V}_{r}^{(0)}-\mathbf{V}_{t}^{(0)}\right) \\
E R R^{(0)}=\iint_{S}\left(\left|F_{P}^{(0)}\right|^{2}+\left|F_{V}^{(0)}\right|^{2}\right) d A \\
n=0
\end{gathered}
$$

Begin loop

$$
\begin{aligned}
& s_{r}^{(n)}=\left[-i \omega \rho_{1} Y_{0}^{1 / 2} \iint_{S} F_{P}^{(n) *} e^{i \mathbf{k}_{1}^{+} \cdot \mathbf{r}} d A\right. \\
& \left.-i Z_{0}^{1 / 2} \iint_{S} \boldsymbol{\nu} \cdot \mathbf{k}_{1}^{+} F_{V}^{(n) *} e^{i \mathbf{k}_{1}^{+} \cdot \mathbf{r}} d A\right]^{*} \\
& s_{t}^{(n)}=\left[-i \omega \rho_{2} Y_{0}^{1 / 2} \iint_{S} F_{P}^{(n) *} e^{i \mathbf{k}_{2}^{-} \cdot \mathbf{r}} d A\right. \\
& \left.+i Z_{0}^{1 / 2} \iint_{S} \boldsymbol{\nu} \cdot \mathbf{k}_{2}^{-} F_{V}^{(n) *} e^{i \mathbf{k}_{2}^{-} \cdot \mathbf{r}} d A\right]^{*} \\
& n=n+1 \\
& A^{(n)}=\iint_{-\infty}^{\infty}\left(\left|s_{r}^{(n-1)}\right|^{2}+\left|s_{t}^{(n-1)}\right|^{2}\right) d k_{x} d k_{y}
\end{aligned}
$$

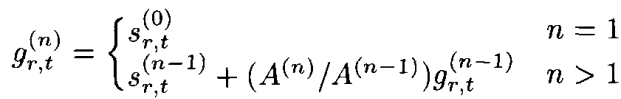

$$
\begin{aligned}
& f_{P}^{(n)}=-i \omega \rho_{1} Y_{0}^{1 / 2} \iint_{-\infty}^{\infty} g_{r}^{(n)} e^{i \mathbf{k}_{1}^{+} \cdot \mathbf{r}} d k_{x} d k_{y} \\
& +i \omega \rho_{2} Y_{0}^{1 / 2} \iint_{-\infty}^{\infty} g_{t}^{(n)} e^{i \mathbf{k}_{2}^{-} \cdot \mathbf{r}} d k_{x} d k_{y} \\
& f_{V}^{(n)}=-i Z_{0}^{1 / 2} \iint_{-\infty}^{\infty} \boldsymbol{\nu} \cdot \mathbf{k}_{1}^{+} g_{r}^{(n)} e^{i \mathbf{k}_{1}^{+} \cdot \mathbf{r}} d k_{x} d k_{y} \\
& +i Z_{0}^{1 / 2} \iint_{-\infty}^{\infty} \boldsymbol{\nu} \cdot \mathbf{k}_{2}^{-} g_{t}^{(n)} e^{i \mathbf{k}_{2}^{-} \cdot \mathbf{r}} d k_{x} d k_{y}
\end{aligned}
$$




$$
\begin{gathered}
B^{(n)}=\iint_{S}\left(\left|f_{P}^{(n)}\right|^{2}+\left|f_{V}^{(n)}\right|^{2}\right) d A \\
\eta^{(n)}=A^{(n)} / B^{(n)} \\
\phi_{r, t}^{(n)}=\phi_{r, t}^{(n-1)}+\eta^{(n)} g_{r, t}^{(n)} \\
F_{P, V}^{(n)}=F_{P, V}^{(n-1)}-\eta^{(n)} f_{P, V}^{(n)} \\
E R R^{(n)}=E R R^{(n-1)}-\eta^{(n)} A^{(n)}
\end{gathered}
$$

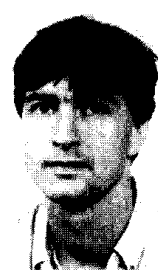

A. P. Berkhoff received the B.Sc. degree in electrical engineering from the Enschede Polytechnic in 1987. From the University of Twente, Enschede, he received the M.Sc. degree in electrical engineering in 1991.

Currently, he is working as a Research Engineer at the University Hospital Nijmegen, where he is engaged in research on computational models for ultrasonic wave propagation in human tissue.

Mr. Berkhoff is a Student Member of the IEEE and a member of the Audio Engineering Society.

End loop

If, after $n=N$ iterations, $E R R^{(n)}$ is small enough, the reflected field $\left\{P_{r}^{(N)}, \mathbf{V}_{r}^{(N)}\right\}$ and the transmitted field $\left\{P_{t}^{(N)}, \mathbf{V}_{t}^{(N)}\right\}$ can be found by substituting $\phi_{r, t}^{(N)}$ in (19) and using (18).

\section{REFERENCES}

[1] W. C. Meecham, "On the use of Kirchhoff approximation for the solution of reflection problems," J. Rational Mech. Anal., vol. 5, no. 2 , pp. 323-333, 1956.

[2] I. A. Urusovskii, "Diffraction of sound on a periodically uneven and inhomogeneous surface," Sov. Phys.-Dokl., vol. 5, pp. 345-348, 1960.

[3] I. A. Urusovskii, "Diffraction by a periodic surface," Sov. Phys.-Acoust., vol. 10, pp. 287-293, 1965.

[4] R. L. Holford, "Scattering of sound waves at a periodic, pressure-release surface: An exact solution," J. Acoust. Soc. Am., vol. 70, pp. 1116-1128, Oct. 1981.

[5] C. T. H. Baker, The Numerical Treatment of Integral Equations. Oxford: Clarendon Press, 1977.

[6] E. I. Thorsos, "The validity of the Kirchhoff approximation for rough surface scattering using a Gaussian roughness spectrum," J. Acoust. Soc. Am., vol. 83, pp. 78-92, January 1988.

[7] P. M. van den Berg, "Reflection by a grating: Rayleigh methods," $J$. Opt. Soc. Am., vol. 10, pp. 1224-1229, Oct. 1981.

[8] D. H. Berman and J. S. Perkins, "Rayleigh method for scattering from random and deterministic interfaces," J. Acoust. Soc. Am., vol. 88, pp. 1032-1044, Aug. 1990.

[9] W. C. Meecham, "Variational method for the calculation of the distribution of energy reflection from a periodic surface I," J. Appl. Phys. vol. 27, pp. 361-367, 1965.

[10] P. M. van den Berg and A. T. de Hoop, "Reflection and transmission of electromagnetic waves at a rough interface between two different media," IEEE Trans. Geosci. and Remote Sensing, vol. GE-22, pp. 42-52, Jan. 1984.

[11] A. I. Nachman, J. F. Smith, and R. C. Waag, "An equation for acoustic propagation in inhomogeneous media with relaxation losses," J. Acoust. Soc. Am., vol. 88, pp. 1584-1595, Sept. 1990 .

[12] J. T. Fokkema and P. M. van den Berg, Seismic Applications of Acoustic Reciprocity. Amsterdam: Elsevier, 1993.

[13] R. F. Millar, "The Rayleigh hypothesis and a related least-squares solution to scattering problems for periodic surfaces and other scatterers," Radio Sci., vol. 8, pp. 785-796, Aug.-Sept. 1973.

[14] A. J. Berkhout, Applied Seismic Wave Theory . Amsterdam: Elsevier, 1987

[15] M. R. Hestenes and E. Stiefel, "Methods of conjugate gradients for solving linear systems," J. Res. Nat. Bur. St. , vol. 49, pp. 409-436, Dec. 1952.

[16] G. H. Golub, C. F. van Loan, Matrix Computations. Baltimore: John Hopkins University Press, 1989

[17] P. M. van den Berg, "Iterative schemes based on minimization of a uniform error criterion," in Application of Conjugate Gradient Method to Electromagnetics and Signal Analysis, PIER 5, T. K. Sarkar, Ed. Amsterdam: Elsevier, 1991, pp. 27-65.

[18] A. P. Berkhoff, P. M. van den Berg, and J. M. Thijssen, "Simulation of wave propagation through aberrating layers of biological media," in Proc. 1994 IEEE Int. Ultrason. Symp., 1994, vol. 3, pp. 1797-1800.

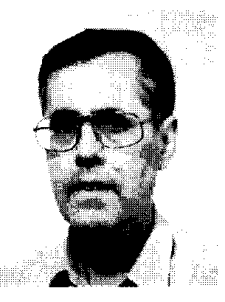

P. M. van den Berg received the degree in electrical engineering from the Polytechnical School of Rotterdam in 1964, the B.S.c. and M.Sc. degrees in electrical engineering, and the Ph.D. degree in technical sciences, all from the Delft University of technology, in 1966, 1968, and 1971, respectively.

From 1967 to 1968, he was employed as a Research Engineer by the Dutch Patent Office. Since 1968, he has been a member of the Scientific Staff of the Electromagnetic Research Group of the Delft University of technology. During these years, he carried out research and taught classes in the area of wave propagation and scattering problems. During the academic year 1973-1974 he was Visiting Lecturer in the Department of Mathematics, University of Dundee, Scotland. During a three-month's period in 1980-1981, he was a Visiting Professor at the Institute of Theoretical Physics, Goteborg, Sweden. He was appointed as a Professor at the Delft University of technology in 1981. At present, his main research interest is the efficient computation of field problems using iterative techniques based on error minimalization, the computation of fields in strongly inhomogeneous media, and the use of wave phenomena in seismic data processing.

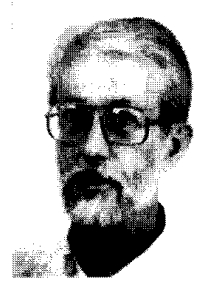

J. M. Thijssen (A'93-M'93-SM'94) received the M.Eng.Sc. degree in technological physics from the Technological University of Delft, Delft, The Netherlands, in 1964, and the Ph.D. degree in experimental physics from the University of Nijmegen, Nijmegen, The Netherlands, in 1969.

From 1964 until 1968 he was a Research Assistant in the Department of Medical physics at the University of Nijmegen. His Ph.D. dissertation was devoted to the differential luminance sensitivity of the human visual system. He continued his in this field with microelectrophysiological studies of the visual system of animals until 1980. In 1968 he became Head of the Biophysics Laboratory of the Department of Opthalmology, University of Nijmegen. In 1969 he started instrumental developments for opthalmological echography, an immersion compound scanner, and more recently he patented a mechanical real-time scanner. In 1975 he received his first grant for research in the field of ultrasonic tissue characterization; this research is continuing. In 1980 he organized the eighth Congress of the International Society for Ultrasound in Opthalmology. From 1982 to 1988 he was the Project Leader of a Concerted Action Programme on Ultrasonic Tissue Characterization of the European Communities. Between 1979 and 1993 he organized nine European Workshops on this topic. He is Cofounder, Past-Secretary/Treasurer of the International Society for Ultrasound in Opthalmology, Member of the National health Council and National Delegate in the European and World Federations for Ultrasound in Medicine and Biology, and in the International Organization for Medical Physics. He is the Founder (1993) and Editor-in-Chief of the European Journal of Ultrasound. He is a member of numerous scientifc organizations and a Senior Member of IEEE. 